\title{
ANÁLISE DOS CUIDADOS DE ENFERMAGEM EM PACIENTES COM LESÕES POR PRESSÃO NA UNIDADE DE TERAPIA INTENSIVA
}

\author{
ANALYSIS OF NURSING CARE IN PATIENTS WITH PRESSURE INJURIES IN \\ THE INTENSIVE THERAPY UNIT
}

\author{
Camila Teixeira de Carvalho Dias, Maria Carolina Salustino dos Santos, Érika Diniz De Oliveria \\ Sousa \\ Centro Universitário de João Pessoa - UNIPÊ
}

\begin{abstract}
To know the nursing care in patients with pressure injury in the ICU. Exploratory-descriptive type, with a quantitative approach. Data collection was performed through the application of a structured questionnaire, containing closed questions, which was applied with 10 nursing team professionals, with 5 nurses and 5 nursing technicians working in a therapy unit Intensive, in a reference Hospital located in João Pessoa-PB, having been applied on weekdays, in the afternoon, in May 2017. Among the results, it was evaluated that the team adequately uses the pressure Injury prevention Protocol, Performing all the care recommended in the patient, and with the elaboration of a plan for the prevention and care of the LPP, however most did not receive training on the protocol of the Ministry of Health's LPP. The provision of nursing care is a necessary factor in the maintenance and recovery.
\end{abstract}

Key words: Injur; Caution; Nursing.
Resumo

Conhecer os cuidados de enfermagem em pacientes com Lesão por Pressão na Unidade de Terapia Intensiva- UTI. Pesquisado tipo exploratório-descritiva, com abordagem quantitativa. A coleta de dados foi realizada mediante a aplicação de um questionário estruturado, contendo questões fechadas, que foi aplicado com 10 profissionais da equipe de enfermagem, sendo 5 enfermeiros e 5 técnicos de enfermagem que atuam em uma Unidade de Terapia Intensiva, em um Hospital de Referência localizado em João Pessoa-PB, tendo sido aplicados em dias úteis, no período da tarde, no mês de Maio de 2017.Dentre os resultados avaliou-se, que a equipe utiliza adequadamente $o$ protocolo de prevenção de Lesões por Pressão, realizando todos os cuidados preconizados no paciente, e com elaboração de plano para a prevenção e cuidados das LPP, no entanto a maioria não recebeu capacitação sobre $o$ protocolo de LPP, do Ministério da Saúde. A prestação de cuidados de enfermagem é fator necessário na manutenção e recuperação do paciente hospitalizado, através das capacitações, sistematização do cuidado e prescrições de enfermagem, é que podemos exercer um assistência de qualidade ao individuo, família $e$ coletividade.

Palavras chave: Lesão; Cuidado; Enfermagem. 


\section{Introdução}

A lesão por pressão (LPP), pode ser definida como um dano localizado na pele e/ou nos tecidos moles subjacentes, que comumente, ocorre sobre uma proeminência óssea ao uso de dispositivo médico ou a outro artefato, esta lesão é decorrente da pressão intensa e/ou combinada com cisalhamento, além disso, a tolerância do tecido mole à pressão e cisalhamento também pode ser afetados pelo microclima, nutrição, perfusão, co-morbidades e condição do tecido mole ${ }^{1}$.

A LPP, é considerada um problema grave e recorrente nos serviços de saúde, que acomete principalmente pacientes hospitalizados, portadores de doenças crônico-degenerativas e idosos. Acredita-se que a grande maioria dessas lesões podem ser prevenidas através de cuidados adequados e qualificados, por uma equipe de saúde orientada e comprometida com a qualidade da assistência prestada, que frequentemente é a equipe de enfermagem ${ }^{2}$.

A lesão por pressão classifica-se em estágio: 10 estágio - pele intacta com eritema não branqueável - em peles de cor escura podem aparece de forma diferente, 20 estágio-perda de espessura parcial da pele com exposição da derme, 3o estágio- apresenta perda total da espessura da pele, 4o estágio- perda total da espessura da pele e perda tissular e o último estágio que é denominado "não classificável", pelo fato de apresentar, perda da pele em sua espessura total e perda tecidual não visível, na qual a base da lesão está coberta por esfacelo ou escara ${ }^{1}$.

Neste sentido, são fatores que contribuem para o desenvolvimento de Lesão por Pressão: a imobilidade, o comprometimento da percepção sensorial ou da cognição, a diminuição da perfusão tissular, a diminuição do status nutricional, o atrito e as forças de tracionamento, o aumento da umidade, bem como as alterações relacionadas à idade ${ }^{3}$.

Estas lesões são geradas pela combinação de fatores intrínsecos e fatores extrínsecos. Dentre os fatores extrínsecos destacam-se: a pressão excessiva sobre os tecidos que devem ser analisados, levando-se em conta a intensidade da pressão, duração e tolerância dos mesmos. Já os intrínsecos apresentam sete fatores, que são: umidade, temperatura, estado geral, idade, mobilidade, peso corpóreo e estado nutricional ${ }^{4}$.

Sendo assim, para avaliar a dimensão e alcance das lesões, é primordial a utilização de protocolos disponibilizados pelo Ministério da
Saúde, juntamente as escalas de avaliação, estudos mostraram que é possível e imprescindível a utilização da escala de predição de risco como forma de avaliar o risco que o paciente possui para desenvolver lesões por pressão ${ }^{5}$.

O uso de protocolos de prevenção e tratamento de Lesões por Pressão é uma ferramenta da sistematização da assistência de enfermagem, que qualifica o cuidado prestado, repercutindo no indicador de qualidade assistencial do trabalho da enfermagem, através da redução da incidência de Lesões por Pressão ${ }^{6}$.

Uma das escalas utilizadas é a de Braden, que foi elaborada com base na fisiopatologia das LPPs, utilizando fatores determinantes, como: a percepção sensorial, umidade da pele, atividades, mobilidade, estado nutricional, fricção e cisalhamento ${ }^{6}$. Diante disso, surgiu o seguinte questionamento: como ocorrem os cuidados de enfermagem de pacientes com lesões por pressão na Unidade de Terapia Intensiva- UTI?

A pesquisa segue tendo por objetivo: avaliar os cuidados de enfermagem em pacientes com Lesão por Pressão na Unidade de Terapia Intensiva- UTI.

A construção desta pesquisa justifica-se pela relevância de publicar a respeito da prevenção e promoção da saúde, é de grande importância observar e investigar soluções e metodologias de melhorar a assistência ao paciente na Unidade de terapia Intensiva, sabendo que o mesmo tem uma maior susceptibilidade de adquirir patologias através do ambiente que está hospitalizado. Há a necessidade de uma assistência de excelência nos serviços de saúde, principalmente a prestação de cuidados realizada pela equipe de enfermagem.

\section{Metodologia}

Pesquisa caracterizada na modalidade exploratório-descritiva, com abordagem quantitativa. Para a construção dos dados, utilizou-se um instrumento de coleta, com metodologia estruturada, contendo questões fechadas, e que foi aplicado com os enfermeiros e técnicos de enfermagem atuantes na Unidade de Terapia Intensiva-UTI, sendo estes 10 profissionais da equipe de enfermagem, 5 técnicos e 5 enfermeiros. A pesquisa ocorreu em um hospital de referência da cidade de João Pessoa- PB, a coleta de dados foi realizada no mês de Maio de 2017.

Foi utilizado o método não probabilístico 
por conveniência, para definição da amostra. Toda pesquisa para ser bem elaborada é necessário estabelecer critérios de participação, para este estudo foram utilizados omo critérios de inclusão: aqueles que estivessem na faixa etária entre 18 e 50 anos (conforme recomendação do Comitê de Ética) e fossem membros da equipe de enfermagem, da Unidade de Terapia Intesiva-UTI do hospital, no ano de 2017, e como critérios de exclusão, profissionaisacima de 50 anos e que não fossem menbros da equipe de enfermagem do setor, no ano de 2017, ou não se inserissem nos critérios de inclusão.

A coleta de dados foi formalizada mediante a aprovação do projeto de pesquisa pelo Comitê de Ética, e o encaminhamento de ofício da coordenação do Curso para a instituição ao local da pesquisa, comunicando a intenção da mesma, além da assinatura destes profissionais, no que se refere ao Termo de Consentimento Livre e Esclarecido (TCLE). este estudo possui a aprovação do cômite de ética do Centro Universitário de João Pessoa UNIPÊ, seguido pelo CAAE: 64815817.9.0000.5176.
A análise dos dados foi realizada com enfoque no método quantitativo, de maneira descritiva, os dados foram apresentados através de gráficos e tabelas, utilizando como ferramenta o software Microsoft Word.

\section{Resultados e Discussões}

Em relação, aos resultados, agora analisados e aplicados com os profissionais de saúde, enfermeiros e técnicos de enfermagem que prestam assistência de enfermagem e realizam cuidados aos pacientes com Lesão por Pressão na UTI, temos os seguintes dados:

\section{Realização da Mudança De Decúbito}

No que se refere ao seguinte questionamento "É realizada a mudança de decúbito a cada duas horas?", temos os seguintes resultados:

Gráfico 1 - Distribuição das respostas dos participantes do estudo $(\mathrm{N}=10)$, de acordo com o questionamento

Gráfico 1 - Distribuição das respostas dos participantes do estudo $(\mathrm{N}=10)$, de acordo com o questionamento:

\section{Mudança de Decúbito}

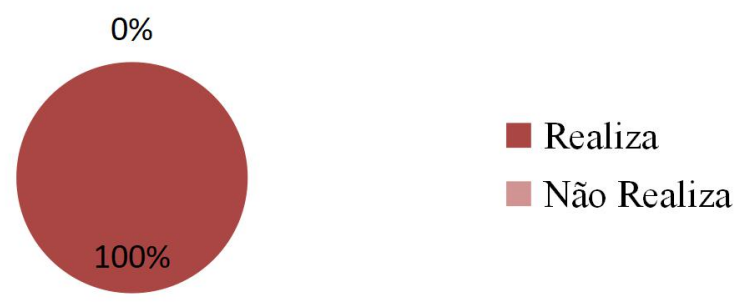

Fonte: Dados da Pesquisa. João Pessoa - PB, Maio, 2017.

De acordo com os dados acima apresentados, podemos perceber que $100 \%$ responderam que realizam a mudança de decúbito. Porém, os enfermeiros relataram que esse reposicionamento é realizado de acordo com a condição e perfil do paciente. 


\section{Instrumento de avaliação para a prevenção de lesão por pressão}

Gráfico 2. Distribuição das respostas dos participantes do estudo $(\mathrm{N}=10)$, de acordo com o questionamento:

\section{Instrumento de Avaliaçao}

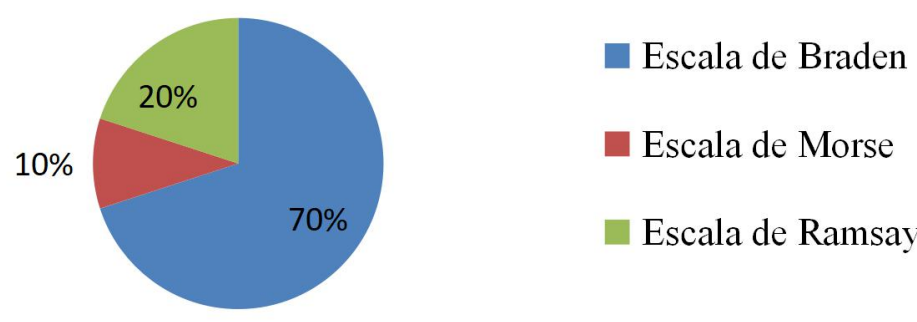

Fonte: Dados da Pesquisa. João Pessoa - PB, Maio, 2017.

Os profissionais de enfermagem $(n=10)$, responderam ao seguinte questionamento: "Qual instrumento de avaliação utilizado para a prevenção de Lesão por Pressão?", seu resultado pode ser observado no gráfico 2 .

Percebe-se que a maioria dos profissionais de enfermagem, ou seja, $70 \%(n=7)$ responderam corretamente, sendo que $10 \%(n=1)$ e $20 \%(n=2)$ marcaram a alternativa errada.
Realização de avaliação de risco referente ao desenvolvimento de lesão por pressão

No que se refere ao questionamento a seguir: "A equipe de enfermagem realiza uma avaliação de risco quando o paciente é admitido na UTI, no que se refere ao desenvolvimento de lesões por pressão ou se já existem lesões instaladas?":

Gráfico 3 - Distribuição das respostas dos participantes do estudo $(\mathrm{N}=10)$, de acordo com o questionamento:

\section{Avaliação de risco}

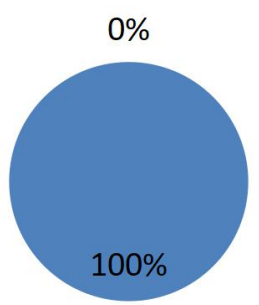

Realiza

Não Realiza

Fonte: Dados da Pesquisa. João Pessoa - PB, Maio, 2017.

Percebe-se que houve uma unanimidade nas respostas, pois $100 \%$ dos participantes do estudo $(\mathrm{N}=10)$, afirmaram que realizavam uma avaliação de risco para o desenvolvimento de lesão por pressão, quando o paciente é admitido na UTI.

\section{Elaboração de plano de cuidados}

No que se refere ao questionamento a seguir: "Uma vez detectado que o paciente apresenta alguma Lesão por pressão, vocês elaboram um plano de cuidados e intervenções para esse paciente?" 
Gráfico 4. Distribuição das respostas dos participantes do estudo $(\mathrm{N}=10)$, de acordo com o questionamento:

\section{Elaboração de Planos de Cuidados e Interveções}

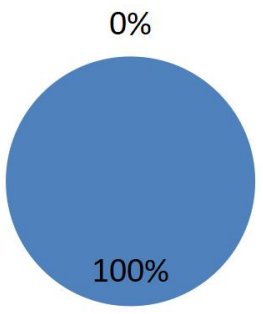

Realiza

Não Realiza

Fonte: Dados da Pesquisa. João Pessoa - PB, Maio, 2017

Dessa forma, podemos perceber que houve uma unanimidade nas respostas, pois $100 \%$ dos participantes do estudo $(\mathrm{N}=10)$, afirmaram que elaboram um plano de cuidados e intervenções para o paciente que já está com uma Lesão por pressão.
Capacitação a respeito do protocolo de prevenção de lesão por pressão do ministério da saúde

Em relação ao seguinte questionamento temos: "Você recebeu alguma capacitação a respeito do protocolo de Lesão por pressão, do Ministério da Saúde?":

Gráfico 5 - Distribuição das respostas dos participantes do estudo $(\mathrm{N}=10)$, de acordo com o questionamento:

\section{Capacitação a Respeito do Protocolo de Prevenção de LPP do Ministerio da Saúde}

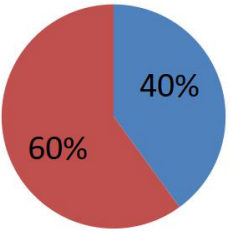

Sim

Não

Fonte: Dados da Pesquisa. João Pessoa - PB, Maio, 2017.

De acordo com os dados apresentados, podemos perceber que $60 \% \quad(n=6)$ dos participantes do estudo afirmaram não terem recebido uma capacitação específica a respeito do protocolo de Lesão por Pressão do Ministério da Saúde e $40 \% \quad(n=4)$ responderam que receberam essa capacitação.

\section{Resultados e discussões}

A Lesão por pressão possui um tratamento minucioso e geralmente prolongado, o que gera um alto custo as instituições e ao governo, como também sobrecarrega o trabalho para a equipe de enfermagem, evidenciando dessa forma a importância de atividades de prevenção ${ }^{6}$.

Portanto, um dos cuidados de enfermagem para a prevenção de lesões por pressão é a mudança de decúbito, que tem por objetivo, modificar o paciente a cada 2 horas, redistribuindo a pressão na pele do mesmo, e assim, reduzir o risco de aparecimento de lesões sobre áreas do corpo vulneráveis. Não é preconizada a frequência com que se deve fazer o reposicionamento, pois a mesma pode ser influenciada por vários fatores relacionados ao indivíduo, como: a tolerância tecidual, nível de atividade e mobilidade, condição clínica global, dentre outros ${ }^{8}$. 
Neste sentido, inclui-se como cuidado da equipe de enfermagem, a avaliação de lesões por pressão mediante algum instrumento utilizado, sendo preconizadas escalas, que mensuram pontos relevantes ao progresso das lesões no paciente.

A escala de Braden é a mais utilizada, na qual caracteriza o paciente sem risco, com risco baixo (15 a 18 pontos), moderado (13 a 14 pontos), alto (10 a 12 pontos) ou muito alto ( $\leq 9$ pontos) para desenvolver Lesões por pressão. É uma escala preditiva, da forma que os seus parâmetros utilizados em associação da avaliação clínica do enfermeiro, identificam e classificam os fatores de risco de maneira inversamente proporcional, ou seja, quanto maior for a soma de pontos, menor será a classificação de risco para ocorrência de lesões ${ }^{2}$.

Portanto, verificou-se que a implantação de um protocolo de prevenção de Lesões por pressão é uma decisão estratégica, para o fortalecimento e melhoria das práticas assistenciais. Dessa forma, nota-se a fundamental importância da efetividade e aplicabilidade de um instrumento de prevenção de lesões por pressão na prescrição de enfermagem ${ }^{7}$. Essa identificação de pacientes em risco é feita através da aplicabilidade de um instrumento validado, que facilita a utilização imediata de medidas preventivas. A avaliação de risco é realizada mediante os seguintes fatores: mobilidade; incontinência; déficit sensitivo e, estado nutricional (incluindo desidratação) ${ }^{8}$.

São cuidados de enfermagem para prevenir lesões por pressão: avaliar, inspecionar a pele do paciente, e prescrever os cuidados específicos para prevenção de alterações cutâneas, sendo que a participação da equipe multiprofissional é fundamental no suporte para a prescrição e no planejamento dos cuidados com o paciente em risco, pois, poderão ser necessários ajustes nutricionais, intervenções para auxiliar a mobilização ou mobilidade dos pacientes, dentre outros $^{9}$.

Porém, foram identificadas algumas fragilidades, onde os profissionais não souberam identificar corretamente qual a escala é utilizada para classificar o risco de Lesão por pressão, assim como, afirmaram não terem recebido uma capacitação a respeito do Protocolo de Prevenção de Lesão por Pressão, do Ministério da Saúde.

Para que o cuidado seja sistematizado e que de maneira eficaz alcance o paciente, é preciso uma educação permanente por parte dos profissionais, é necessário conhecer e embasarse cientificamente para cuidar do ser humano. A equipe de enfermagem de maneira imprescindível precisa estar inserida em capacitações e atualidades, para que assim possam prestar uma assistência de qualidade ${ }^{10}$.

\section{Conclusão}

Mediante pesquisa desenvolvida, ao longo de toda a elaboração deste artigo, foi possível observar o quanto é impresncidivel um plano de cuidados elaborado pela enfermagem para os pacientes com lesão por pressão.

No que se referem às questões norteadoras do estudo, os resultados foram bastante positivos. Verificou-se que a equipe utiliza adequadamente o protocolo de prevenção de Lesões por Pressão, realizando todos os cuidados preconizados nesse paciente, para a prevenção e cuidados da Lesão por pressão. Constatei também que existia um fluxograma deste protocolo anexado no quadro de avisos da UTI, o que é extremamente relevante, demonstrando que os profissionais de fato conhecem e aplicam as orientações fornecidas no mesmo.

No entanto, foram identificadas algumas fragilidades, onde $30 \%$ dos profissionais não souberam identificar corretamente a escala que seria utilizada para classificar o risco de uma Lesão por pressão, assim como grande parte dos entrevistados afirmou que, não receberam nenhuma capacitação a respeito do Protocolo de Prevenção de Lesão por Pressão, do Ministério da Saúde.

É extremamente importante que haja um investimento na educação permanente em saúde, para que os profissionais possam se capacitar e se atualizar diante das mudanças de nomenclatura e da classificação destas lesões, pois isso poderá interferir diretamente na qualidade da assistência prestada, garantindo grandes benefícios aos pacientes, além de reduzir os gastos na instituição.

\section{Referências}

1. NationalPressureUlcerAdvisoryPanel (NPUAP) Anuncia uma Mudança na Terminologia de Úlcera de Pressão a Lesão Pressão e Atualiza os Estágios de Lesão Pressão.2016. Disponível em: http://www.npuap.org/national-pressure-ulceradvisory-panel-npuap-announces-a-change-interminology-from-pressure-ulcer-to-pressureinjury-and-updates-the-stages-of-pressureinjury/

2. Borghardt AT, Prado TN, Araújo TM,Rogenski NMB, Bringuente MEO. Avaliação 
das escalas de risco para úlcera por pressão em pacientes críticos: uma coorte prospectiva.Rev. Latino-Am. Enfermagem[Internet]. 2015jan-fev [Citado 07 de Dez. de 2017] ;23(1):28-35. Disponível em: http://www.scielo.br/pdf/rlae/v23n1/pt 01041169-rlae-23-01-00028.pdf

3. Smeltzer SC,BareG. Brunner\&Suddarth Tratado de Enfermagem Médico-Cirúrgica. 12a. ed. Rio de Janeiro: Editora GuanabaraKoogan: S.A; 2011.

4. Quirino DES, Faustino AM, Freitas RO, Oliveira AB,Medved IV. Fatores de Risco para o Desenvolvimento de Úlcera por Pressão em Unidade de Internação Clínica.Rev. Estima[Internet] . 2014 [Citado em 06 de Dez. de 2017];12 ( 4). Disponível em: https://www.revistaestima.com.br/index.php/esti ma/article/view/99

5. Medeiros ABF,Lopes CHAF, Jorge BAS. Análise da prevenção e tratamento das úlceras por pressão propostos por enfermeiros. RevEscEnferm [Internet]. 2009 [Citado em 07 de Dez. de 2017]; 43 (1):223-228. Disponível em : http://www.scielo.br/pdf/reeusp/v43n1/29. pdf

6. Saatkamp F. Cuidados de Enfermagem na Prevenção às Ulceras por Pressão em Pacientes Hospitalizados. Lajeado: Univates; $2012 . \quad$ Disponível em:https://www.univates.br/bdu/bitstream/107 37/412/1/FernandaSaatkamp.pdf

7. Silva EWNL, Araújo RA, Oliveira EC, Falcão VTFL. Aplicabilidade do protocolo de prevenção de úlcera de pressão em unidade de terapia intensiva. Rev. bras. ter. intensiva [ Internet]. 2010 abr-jun [Citado em 08 de Dez. de 2017] ;22(2): 175-185. Disponível em: http://www.scielo.br/pdf/rbti/v22n2/a12v22n2.p df 8. BRASIL. Protocolo para Prevenção de Úlcera por Pressão.Brasília:Ministério da Saúde, Anvisa, Fiocruz. 2013. Disponível em: http://www.hospitalsantalucinda.com.br/downlo ads/prot_prevencao ulcera por_pressao.pdf 9. Anselml ML,PeduzziM, Junior Fl. Incidence of pressure ulcer and nursing interventions. Acta Paul Enferm. [Internet] 2009 mar-jul[Citado em 07 de Dez. de 2017]; 22 (3)200:257-264. Disponível em: http://www.scielo.br/pdf/ape/v22n3/a04v22n3 10. Santos CT, Oliveira MC, Pereira AGS, Suzuki LM, Lucena AF. Indicador de qualidade assistencial úlcera por pressão: análise do prontuário e de notificação de incidente. rev. Gaúcha de enfermagem. [Internet] 2013 [Citado em 08 de Dez. de 2017]; 34(1): 111-
118.Disponível

em: https://www.lume.ufrgs.br/bitstream/handle/10 183/85388/000879360.pdf?sequence $=1$

\section{Endereço para Correspondência}

Centro universitário de João Pessoa - UNIPÊ

Rodovia BR-230,km 22, s/n - Água Fria, João Pessoa - PB, 58053-000

e-mail: camilatcs2@gmail.com

Recebido em 19/12/2017

Aprovado em 01/11/2018

Publicado em 11/03/2019 